ORIGINAL ARTICLE

\title{
Effect of blood transfusions on oxidative stress in preterm infants
}

\author{
C Dani, E Martelli, G Bertini, M Pezzati, M Rossetti, G Buonocore, P Paffetti, F F Rubaltelli
}

Arch Dis Child Fetal Neonatal Ed 2004;89:F408-F411. doi: 10.1136/adc.2003.037085

See end of article for authors' affiliations

....................

Correspondence to: Professor Dani, Division of Neonatology, Careggi University Hospital, University of Florence School of Medicine, Viale Morgagni, 85 Firenze, Italy; cdani@.unifi.lt

Accepted 23 October 2003

Objective: To confirm the increase in non-transferrin bound iron (NTBI) after packed red cell (PRC) transfusion and to evaluate the association with increased oxidative stress in preterm infants.

Method: Twenty healthy preterm infants (gestational age 28.2 (2.2) weeks; birth weight 1047 (230) g), who required blood transfusion for anaemia of prematurity were prospectively studied. Serum concentrations of NTBI, total hydroperoxides $(\mathrm{TH})$, and protein $\mathrm{SH}$ groups, and plasma total radical trapping antioxidant capability (TAC) were measured within three hours before and after PRC transfusion. The infants were transfused with 38.6 (23) $\mathrm{ml} \mathrm{PRCs} \mathrm{over} 5.8$ (1.0) hours, at a mean age of 43.3 (25.1) days.

Results: After PRC transfusion, haemoglobin concentration increased from 9.2 (1.1) to 14.6 (1.5) g/l. Mean plasma NTBI concentration after transfusion was significantly higher $(0.43(0.45) \vee 2.03$ (1.31) $\mu \mathrm{mol} / \mathrm{l} ; \mathrm{p}=0.001$ ), while plasma concentrations of TH (212.3 (42.2) v 214.7 (66.3) Carr units/l) and protein SH groups (317.5 (38.8) v 353.8 (57.4) $\mu \mathrm{mol} /$ ), and TAC (256.3 (36.1) v 267.1 (42.4) $\mu \mathrm{mol}$ $\mathrm{HClO} / \mathrm{ml}$ ) remained unchanged.

Conclusion: For three hours after PRC transfusion, plasma NTBI is significantly increased in preterm infants, but this is not associated with significant changes in oxidative stress.

B lood transfusion has been suggested to be a risk factor for the development of retinopathy of prematurity $(\mathrm{ROP})^{1-4}$ and chronic lung disease (CLD)..$^{5-7}$ Although unproved, the possible mechanism by which blood transfusions may contribute to the development of ROP and CLD is the increase in oxygen delivery to the retina and lung, secondary to increased packed cell volume and lower oxygen affinity of adult haemoglobin in packed red cells (PRCs), ${ }^{1}$ and secondary iron overload: transfused cells have a shortened half life, and the iron they contain may exert a pro-oxidant effect. ${ }^{8}$ We recently found that PRCs contain about $0.5 \mathrm{mg}$ iron $/ \mathrm{ml}$. Therefore few transfusions can dramatically increase body iron during the first weeks of life. ${ }^{2}$ On the other hand, Hirano et $_{\text {al }}{ }^{8}$ showed that plasma non-transferrin bound iron (NTBI) is significantly increased after blood transfusion in preterm infants and that some of it exists in its pro-oxidant ferrous form. However, it has not been confirmed that NTBI is actually capable of catalysing the formation of reactive oxygen species in vivo and inducing oxidative stress in preterm infants.

Thus we hypothesised that free iron given through blood transfusions may induce oxidative stress in preterm infants. To assess this hypothesis, the plasma concentration of NTBI and, as markers of oxidative stress, total hydroperoxides (TH) and protein $\mathrm{SH}$ groups, and the plasma total antioxidant capacity (TAC) were measured in a cohort of preterm newborns before and after blood transfusion.

\section{MATERIALS AND METHODS Patients}

With the approval of the local ethics committee and once informed parental consent had been obtained, we prospectively studied infants with a gestational age $<32$ weeks and birth weight appropriate for gestational age, who were transfused because of anaemia of prematurity after the first week of life. Infants with major congenital malformations were excluded.
Blood transfusions were performed using adult PRCs at an infusion rate of $5 \mathrm{ml} / \mathrm{kg} / \mathrm{h}$. PRC transfusions were performed when the packed cell volume was $<40 \%$ in infants with severe cardiopulmonary disease, when the packed cell volume was $<30 \%$ in infants with moderate cardiopulmonary disease, and when packed cell volume was $<25 \%$ in infants with symptomatic anaemia.

For each patient, birth weight, gestational age, Apgar score, antenatal steroid treatment, age at blood transfusion, and amount of PRCs were documented. In addition, the occurrence of CLD (defined as oxygen requirement at 36 weeks of postconceptional age) and ROP were recorded. ROP was classified according to the International Classification of Retinopathy of Prematurity.

Heparinised blood samples of $1.5 \mathrm{ml}$ were collected within three hours before and after PRC transfusion for the measurement of plasma concentrations of NTBI, TH, and protein $\mathrm{SH}$ groups, and TAC.

\section{Procedures}

Blood samples were immediately centrifuged (1000 g, $10 \mathrm{~min})$. The plasma was stored in plastic metal free containers at $-80^{\circ} \mathrm{C}$ until analysis.

Plasma NTBI concentrations were determined by high performance liquid chromatography (HPLC) by the partially modified method of Kime et al. ${ }^{10}$ The system was operated isocratically at a pressure of about $1.15 \mathrm{MPa}$ and flow rate of $0.75 \mathrm{ml} / \mathrm{min}$. The detection wavelength was $450 \mathrm{~nm}$ with a reference wavelength at $620 \mathrm{~nm}$. A low affinity ligand, disodium nitriloacetic acid, was first used to complex all low molecular mass iron and iron non-specifically bound to serum proteins such as albumin. As it does not remove iron bound to transferrin or ferritin, a two step filtration process was used: a $100 \mathrm{kDa}$ molecular mass cut off Whatman

Abbreviations: CLD, chronic lung disease, NTBI, non-transferrin bound iron; PRC, packed red cell; ROP, retinopathy of prematurity; TAC, total radical trapping antioxidant capability; $\mathrm{TH}$, total hydroperoxides 
ultracentrifuge filter followed by a $20 \mathrm{kDa}$ cut off filter. The filtrate was analysed by direct injection into a reverse phase HPLC system, using precolumn derivatisation with the high affinity iron chelator CP22 (3-hydroxyl-1-propyl-2-methylpyridin-4-one hydrochloride).

$\mathrm{TH}$ represents a measure of overall oxidative stress, given that they are the intermediate oxidative products of lipids, peptides, and amino acids. TH production was measured with a d-ROMs Kit (Diacron, Rome, Italy) as described by Buonocore et al. ${ }^{11}$ This method makes it possible to estimate $\mathrm{TH}$ present in a $10 \mu \mathrm{l}$ blood sample using a spectrophotometric procedure. Hydroperoxidic groups are attacked by the iron, decompartmentalised from the transport protein in $1 \mathrm{ml}$ acetate buffer at a $\mathrm{pH} 4.8$, to catalyse formation of reactive oxygen metabolite by Fenton's reaction. The peroxy and alkoxy radicals produced, the quantities of which are directly proportional to peroxides present in the plasma, are trapped chemically by $10 \mu \mathrm{l}$ chromogen (N,N-diethyl p-phenyldiamine) in an electron transfer process leading to the formation of the radical cation of this chromogen. The purple colour resulting from this reaction over time was then monitored in a UV-VIS spectrophotometer ( $\lambda$ 16; PerkinElmer, Norwalk, Connecticut, USA) at $505 \mathrm{~nm}$. The results were expressed in conventional units, called Carr units; I Carr unit is equal to a concentration of $0.08 \mathrm{mg} / \mathrm{l}$ hydrogen peroxide. TAC was measured with an OXY-Adsorbent Test (Diacron) as described by Trotti et al. ${ }^{12}$ This method is based on the capacity of a massive dose of $\mathrm{HClO}$ to oxidise the physiological antioxidant reef (uric acid, GSH, thiol groups, vitamins, glutathione peroxidase, superoxide dismutase, catalase, etc.). The efficacy of the antioxidant system can be monitored indirectly by measuring the excess of $\mathrm{HClO}$ in the serum. As HClO reacts with a correctly buffered chromogenic substrate ( $\mathrm{N}, \mathrm{N}$-diethyl p-phenyldiamine), a coloured complex develops which can be measured photometrically, presenting a maximum peak of absorbance at 505 or $546 \mathrm{~nm}$. The concentration of the coloured complex is directly proportional to the concentration of HClO and indirectly to the antioxidant capacity. A $10 \mu \mathrm{l}$ blood sample, previously diluted 1:100 with distilled water, must be added and mixed with $1 \mathrm{ml}$ oxidant solution and $10 \mu \mathrm{l}$ chromogen. The purple colour resulting from this reaction over time is then monitored in a UV-VIS spectrophotometer at 505 or $546 \mathrm{~nm}$. For each series of assays, a standard with an assigned value, previously diluted 1:100 with distilled water as for the samples, and a blank reagent, obtained by replacing serum with distilled water, were included. The absorbances were measured immediately. The absorbance of the reagent blank was subtracted from those of the standard and samples. The antioxidant capacity, expressed as $\mu \mathrm{mol} \mathrm{HClO} /$ $\mathrm{ml}$ serum, was calculated from the following formula:

$$
\begin{aligned}
& \left(\left(\mathrm{Abs}_{\text {Blank }}-\mathrm{Abs}_{\text {Sample }}\right) /\left(\mathrm{Abs}_{\text {Blank }}-\mathrm{Abs}_{\text {Standard }}\right)\right) \times \\
& {[\text { Standards }]}
\end{aligned}
$$

where Abs is absorbance.

Protein SH groups were measured with a SHp Test (Diacron) using the spectrophotometric procedure of Ellman. ${ }^{13}$ This method is based on the capacity of protein SH groups present in the sample to bind a chromogen $(5,5-$ dithiobis-2-nitrobenzoic acid). A $1 \mathrm{ml}$ sample must be mixed with $20 \mu \mathrm{l}$ chromogen. The yellow colour resulting from this reaction over time is then monitored in a UV-VIS spectrophotometer at $405 \mathrm{~nm}$. For each series of assays, a standard solution of $496 \mu \mathrm{mol} / \mathrm{l} \mathrm{SH}$ groups was prepared. The SH group concentration, expressed as $\mu \mathrm{mol} / \mathrm{l}$, was calculated from the following formula:

$$
\begin{aligned}
& \left(\mathrm{Abs}_{\text {Sample }}-\left(\mathrm{Abs}_{\text {Sample Blank }}+\mathrm{Abs}_{\text {Reagent Blank }}\right) /\right. \\
& \left.\left(\mathrm{Abs}_{\text {Standard Blank }}+\mathrm{Abs} \text { Reagent Blank }\right)\right) \times 496
\end{aligned}
$$

where Abs is absorbance and 496 is the standard SH group concentration.

\section{Statistical analysis}

In planning our study, we calculated that a sample size of at least 18 infants in each group was required to detect a difference of $30 \%$ in plasma $\mathrm{TH}$ concentration after blood transfusion, with $80 \%$ power at 0.05 concentration.

The data are expressed as mean (SD). Analysis was performed using analysis of variance for continuous variables or the Wilcoxon rank sum test for non-parametric data. Simple regression analysis was used to assess the correlation between NTBI, TH, TAC, and plasma protein SH group concentrations. $p<0.05$ was considered significant.

\section{RESULTS}

We studied 20 preterm infants (gestational age 28.2 (2.2) weeks; birth weight 1047 (230) g; 13 boys) who were all treated with antenatal steroids. Twelve infants had respiratory distress syndrome, seven had transient tachypnoea, and one had persistent pulmonary hypertension. All required oxygen: 12 were mechanically ventilated (10 high frequency oscillatory ventilation, two synchronised intermittent positive pressure ventilation), and eight were treated with nasal continuous positive airway pressure. None of our patients were ventilated or oxygen dependent at the time of study, and all received full enteral feeding. Three (15\%) developed CLD, and eight (40\%) developed ROP (seven cases of stage l ROP, one case of stage 2 ROP).

All enrolled infants received at least one PRC transfusion (12 received one transfusion, and eight received two transfusions) before the study, but this occurred at least 10 days before the study started.

The mean volume of PRCs transfused was 38.6 (23) ml over 5.8 (1.0) hours at a mean age of 45.5 (23) days of life. After transfusion, haemoglobin concentration increased from 9.2 (1.1) to $14.6(1.5) \mathrm{g} / \mathrm{l}$, and packed cell volume increased from 28.6 (3.4) to 44.7 (4.4).

Before the transfusion, NTBI was detected in $65 \%(13 / 20)$ of our patients, and after the transfusion NTBI was detected in $95 \%(19 / 20)$ of our patients. Only one patient did not have NTBI before or after the transfusion. The mean plasma NTBI concentration after transfusion was significantly higher than before $(0.43$ (0.45) $v 2.03$ (1.31) $\mu \mathrm{mol} / \mathrm{l} ; \mathrm{p}<0.001)$ (fig l). Plasma NTBI concentration did not correlate with TH $(r=0.2912, \mathrm{p}>0.05)$, protein SH groups $(r=-0.3056$, $\mathrm{p}>0.05)$, or TAC $(r=-0.2804, \mathrm{p}>0.05)$.

The mean plasma concentrations of TH (212.3 (42.2) $v$ 214.7 (66.3) Carr units/l) and protein SH groups (317.5 (38.8) v $353.8(57.4) \mu \mathrm{mol} / \mathrm{l}$ ) and mean TAC (256.3 (36.1) $v$ 267.1 (42.4) $\mu \mathrm{mol} \mathrm{HClO} / \mathrm{ml})$ had not changed after transfusion (fig 2). A significant correlation was observed between $\mathrm{TH}$ and TAC, between $\mathrm{TH}$ and protein $\mathrm{SH}$ groups, and between TAC and protein SH groups, both before and after transfusion (fig 3).

\section{DISCUSSION}

Several authors ${ }^{1-6}$ have suggested that a possible mechanism by which blood transfusions may cause the development of CLD and ROP in preterm infants is secondary iron overload.

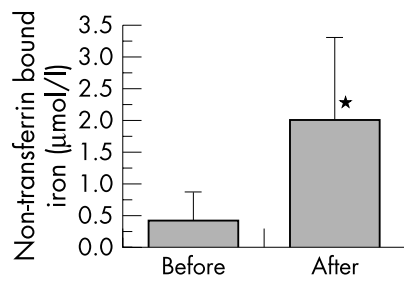

Figure 1 Plasma concentration of non-transferrin bound iron before and after packed red celltransfusion. *Significantly different from before transfusion, $p=0.001$. 


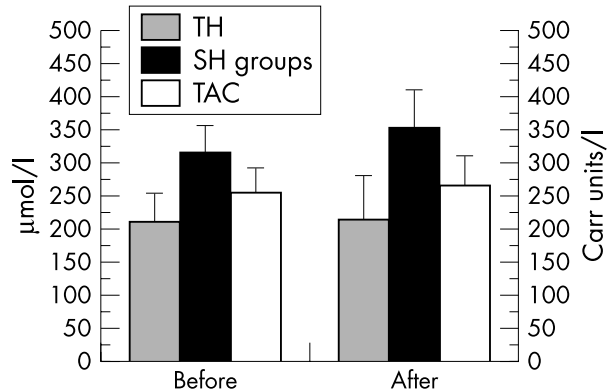

Figure 2 Changes in total hydroperoxides (TH) (Carr units/l), protein $\mathrm{SH}$ groups $(\mu \mathrm{mol} / \mathrm{l})$, and total antioxidant capability (TAC) ( $\mu \mathrm{mol} / \mathrm{l})$ before and after packed red cell transfusion.

Increased free iron may catalyse Fenton reactions, which produce free hydroxyl radicals ${ }^{14}$ from superoxide and hydrogen peroxide capable of damaging the lungs and the retina. Normally, protection against free iron is provided by caeruloplasmin (which converts the pro-oxidant ferrous iron into the ferric state) and transferrin (which binds the ferric iron), but in preterm infants less than 33 weeks gestation, the concentrations of these binding proteins are low, ${ }^{5}{ }^{15}$ and rapid saturation of transferrin occurs. ${ }^{16}$ This hypothesis has not been confirmed in vivo.

Recently, Wardle et $a l^{17}$ found an increase in urinary malondialdehyde 24 hours after blood transfusion and interpreted this as evidence of increased lipid peroxidation. Unfortunately, urinary aldehyde excretion is influenced by numerous factors that affect the formation of lipid peroxides in vivo such as energy status, physical activity, and environmental temperature, as well as by variations in the intake of peroxides in the diet and parenteral fluid..$^{18}$ Thus, because of the limits of the method used, the data of Wardle et al cannot conclusively show the association between PRC transfusion and lipid peroxidation.

Our study, in agreement with Hirano et al, ${ }^{5}$ confirms that NTBI significantly increases after blood transfusions, and shows that this occurs within the first three hours and not only after 24 hours as previously reported. We also found that 13 of the 20 patients studied had NTBI even before blood transfusion, which is consistent with other reports. ${ }^{51019}$ This probably occurs because of the low ferroxidase activity-
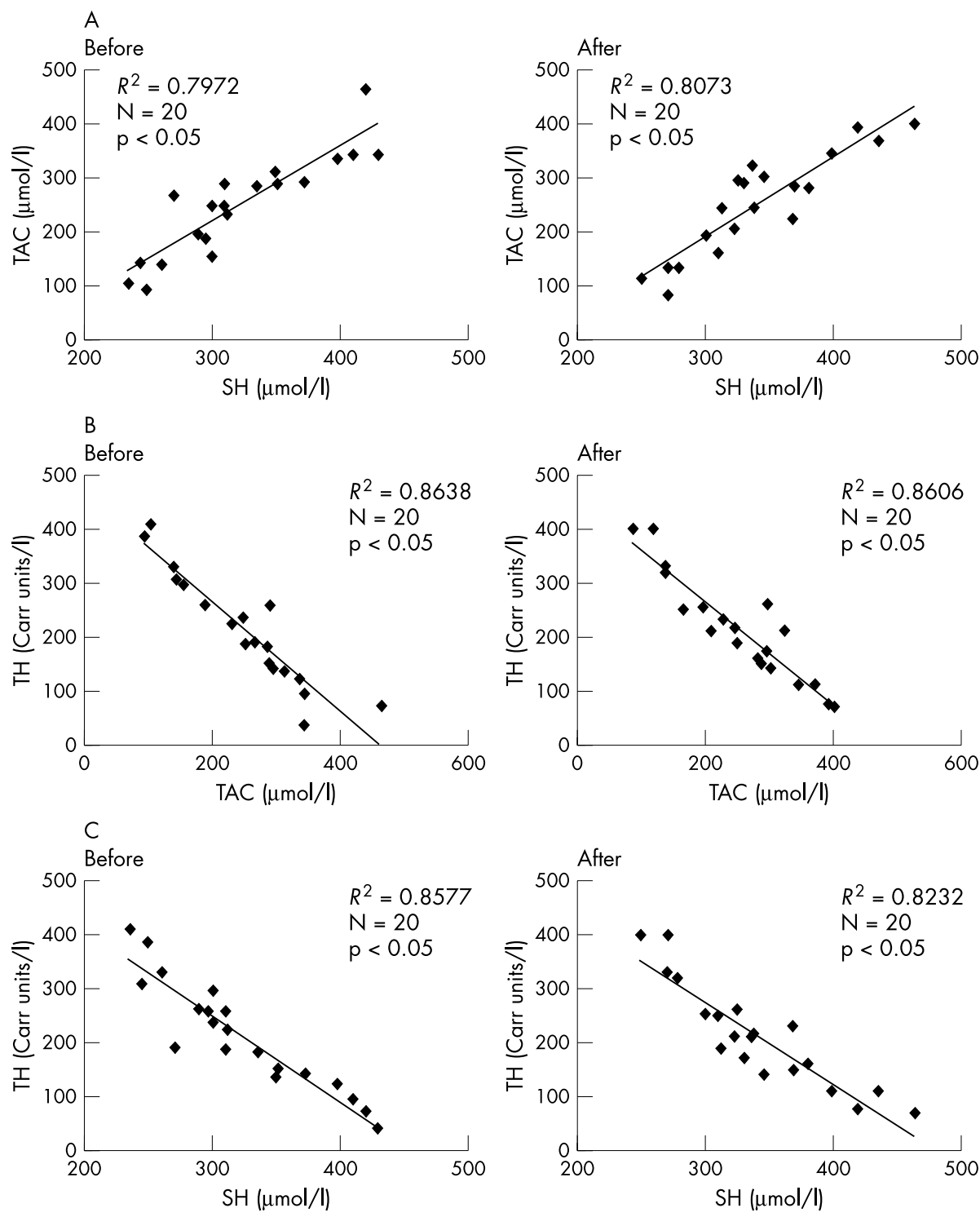

Figure 3 Correlation between plasma total antioxidant capacity of plasma (TAC) and protein SH groups (SH) (A), total hydroperoxide concentration (TH) and TAC (B), and TH and SH (C), both before and after blood transfusion. 
that is, caeruloplasmin activity-and the reduction of ferric iron to its ferrous form by ascorbic acid. ${ }^{5}$

In our study, we found that, during the first three hours after PRC transfusion, oxidative stress does not increase. This suggests that the increase in NTBI after transfusion is not sufficient to induce oxidative stress in our patients, as confirmed by the lack of correlation between NTBI and the oxidative stress markers. Furthermore, it does not support the hypothesis ${ }^{5}{ }^{17}$ that preterm infants should be transfused with whole blood as an alternative to PRC to limit oxidative damage.

To interpret the results of our study, it can be hypothesised that our patients were studied too early after the transfusion and that free iron needs longer than three hours to induce the production of free hydroxyl radicals by the Fenton reaction. This is unlikely because oxygen free radicals start to be produced as soon as chemical conditions are suitable. On the other hand, it cannot be excluded that oxidative stress occurred immediately after the transfusion and that three hours later it had disappeared; however, in that case the oxidative stress would not be clinically relevant. Another hypothesis is that our patients were old enough (34.7 (5.5) weeks of postconceptional age) to reach a plasma caeruloplasmin concentration sufficient to reduce free iron activity and to prevent the production of oxygen derived free radicals despite the overload of NTBI, as occurs in full term infants. ${ }^{5}$ Unfortunately, the small volume of the blood samples from our patients did not allow us to confirm or refute this possibility by measuring plasma caeruloplasmin concentration and plasma ferroxidase activity.

In conclusion, we have shown that three hours after blood transfusion, plasma NTBI concentration is significantly increased in preterm infants, but this increase is not associated with an increase in oxidative stress. These data are reassuring that PRC transfusion does not increase the risk of oxidative stress in preterm infants, but cannot be extrapolated to extremely low birthweight infants and infants who receive multiple close transfusions.

\section{Authors' affiliations}

C Dani, E Martelli, G Bertini, M Pezzati, F F Rubaltelli, Division of Neonatology, Careggi University Hospital, Florence, Italy
M Rossetti, Central Laboratory of Chemical-Clinical Analysis, Careggi University Hospital

G Buonocore, P Paffetti, Department of Pediatrics, Obstetrics and Reproductive Medicine, University of Siena, Siena, Italy

\section{REFERENCES}

1 Cooke RWI, Clark D, Nickey-Dwyer M, et al. The apparent role of blood transfusion in the development of retinopathy of prematurity. Eur J Pediatr 1993; 152:833-6.

2 Dani C, Reali MF, Bertini G, et al. The role of blood transfusions and iron intake on retinopathy of prematurity. Early Hum Dev 2001;62:57-63.

3 Hesse L, Eberl M, Schlaud C, et al. Blood transfusion. Iron load and retinopathy of prematurity. Eur J Pediatr 1997;156:465-70.

4 Inder TE, Clemett RS, Austin NC, et al. High iron status in very low birth weight infants is associated with an increased risk of retinopathy of prematurity. J Pediatr 1997; 131:541-4.

5 Hirano K, Morinobu T, Kim H, et al. Blood transfusions increases radical promoting non-transferrin bound iron in preterm infants. Arch Dis Child Fetal Neonatal Ed 2001;84:F188-93.

6 Silvers KM, Gibson AT, Russell JM, et al. Antioxidant activity, packed cell transfusions, and outcome in premature infants. Arch Dis Child Fetal Neonatal Ed 1998;78:F214-19.

7 Cooke RWI, Drury JA, Yoxall CW. Blood transfusion and chronic lung disease in preterm infants. Eur J Pediatr 1997;156:47-50.

8 Bard H, Widness JA. The life span of erythrocytes transfused to preterm infants. Pediatr Res 1997;42:9-11.

9 Committee for the classification of retinopathy of prematurity. An international classification of retinopathy of prematurity. Pediatrics 1984;74:127-33.

10 Kime R, Gibson A, Yong W, et al. Chromatographic method for the determination of non-transferrin-bound iron suitable for use on the plasma and bronchoalveolar lavage fluid of preterm babies. Clin Sci 1996:91:633-8.

11 Buonocore G, Perrone S, Longini M, et al. Total hydroperoxide and advanced oxidation protein products in preterm hypoxic babies. Pediatr Res 2000;47:221-4.

12 Trotti R, Carratelli M, Barbieri $M$, et al. Oxidative stress and a thrombophilic condition in alcoholics without severe liver disease. Hematologica 2001;86:85-91.

13 Ellman GL. Tissue sulfhydryl groups. Arch Biochem Biophys 1959;82:70-7.

14 Gutteridge JMC. Fate of oxygen free radicals in extracellular fluids. Biochem Soc Trans 1982; 10:72-3.

15 Lackman GM, Schnieder C, Bohner J. Gestational age-dependent reference values for iron and selected proteins of iron metabolism in serum premature human neonates. Biol Neonate 1998;74:208-13.

16 Siimes MA, Addiego JE, Dallman PR. Ferritin in serum, diagnosis of iron deficiency and overload in infants and children. Blood 1974;43:581-90.

17 Wardle SP, Drury J, Garr, et al. Effect of blood transfusion on lipid peroxidation in preterm infants. Arch Dis Child Fetal Neonatal Ed 2002;86:F46-8.

18 Draper HH, Csallany AS, Hadley M. Urinary aldehydes as indicators of lipid peroxidation in vivo. Free Radic Biol Med 2000;29:1071-7.

19 Evans PJ, Evans R, Kovar IZ. Bleomycin-detectable iron in the plasma of premature and full-term neonates. FEBS Lett 1992;303:210-12. 